\title{
Diversite Phlebotomienne Dans Trois Villages De La Commune De Bouake (Côte D'ivoire) Durant La Periode De Mars A Juin 2019
}

Assovié Koco Rita Nadège, PhD student

Centre d'Entomologie Médicale et Vétérinaire

Université Alassane Ouattara, Bouaké, Côte d'Ivoire

Djohan Vincent, PhD

Atta Angui Amoikon Frédéric, PhD

Aké Donald Hermann Arnaud, PhD student

Aka Tigori Armel, PhD student

Unité de Formation et de Recherche Sciences pharmaceutiques et biologiques, Université Felix Houphouët- Boigny, Abidjan, Côte d'Ivoire

Akoliba Koliba Patrice, entomological technician

Institut Pierre Richet, Bouaké,

Institut National de Santé Publique, Abidjan Côte d'Ivoire

Djakaridja Berté, PhD student

Ta Bi Tra Dieudonné, PhD student

Laboratoire de Zoologie et Biologie Animale, UFR Biosciences,

Université Félix Houphouët-Boigny, Abidjan, Côte d'Ivoire

Kaba Dramane, PhD

Institut Pierre Richet, Bouaké,

Institut National de Santé Publique, Abidjan, Côte d'Ivoire

Yapi Yapi Grégoire, PhD

Centre d'Entomologie Médicale et Vétérinaire

Université Alassane Ouattara, Bouaké, Côte d'Ivoire

Doi:10.19044/esj.2020.v16n12p224 URL:http://dx.doi.org/10.19044/esj.2020.v16n12p224

Résumé

La leishmaniose cutanée est une parasitose due à des protozoaires du genre Leishmania et répandue dans le monde. Une enquête médicale réalisée dans trois villages de la ville de Bouaké, autour de cas suspects de leishmaniose cutanée, a permis de confirmer la présence de cette pathologie dans ces villages. Dans le cadre de l'identification des acteurs du cycle épidémiologique de la leishmaniose cutanée, une étude entomologique a été conduite dans ces villages afin d'identifier les phlébotomes potentiels vecteurs 
de leishmanies. Les pièges huileux et les pièges lumineux CDC, ont été posés entre mars et juin 2019, suivant une méthode de rotation entre les sites de piégeage. Ils ont été posés entre $17 \mathrm{~h}$ et $18 \mathrm{~h}$ et relevés le lendemain matin entre $7 \mathrm{~h}$ et $8 \mathrm{~h}$. La diversité spécifique des espèces identifiées a été déterminée à partir des indices écologiques d'équitabilité et de Hill. Ces pièges ont permis la capture de 135 phlébotomes, dont 78 ont été morphologiquement identifiés. Le genre Sergentomyia constituait $91 \%$ de nos récoltes contre $9 \%$ pour le genre Phlebotomus. Ph. bergeroti, Ph. rodhaini et Ph. sergenti étaient les espèces du genre Phlebotomus, capturées dans ces sites. Des travaux approfondis portant sur l'identification du parasite à la fois chez les phlébotomes et chez l'homme doivent être effectués, afin d'identifier les phlébotomes incriminés dans la transmission des leishmanies à Bouaké.

Mots-clés : Leishmaniose cutanée, Phlebotomus, Sergentomyia, Bouaké, Côte d'Ivoire 


\title{
Phlebotomic Diversity in Three Villages of the Commune De Bouake (Côte d'Ivoire) During the Period from March to June 2019
}

Assovié Koco Rita Nadège, PhD student

Centre d'Entomologie Médicale et Vétérinaire

Université Alassane Ouattara, Bouaké, Côte d'Ivoire

Djohan Vincent, PhD

Atta Angui Amoikon Frédéric, PhD

Aké Donald Hermann Arnaud, PhD student

Aka Tigori Armel, PhD student

Unité de Formation et de Recherche Sciences pharmaceutiques et biologiques, Université Felix Houphouët- Boigny, Abidjan, Côte d'Ivoire

Akoliba Koliba Patrice, entomological technician

Institut Pierre Richet, Bouaké,

Institut National de Santé Publique, Abidjan Côte d'Ivoire

Djakaridja Berté, PhD student

Ta Bi Tra Dieudonné, PhD student

Laboratoire de Zoologie et Biologie Animale, UFR Biosciences,

Université Félix Houphouët-Boigny, Abidjan, Côte d'Ivoire

Kaba Dramane, PhD

Institut Pierre Richet, Bouaké,

Institut National de Santé Publique, Abidjan, Côte d'Ivoire

Yapi Yapi Grégoire, PhD

Centre d'Entomologie Médicale et Vétérinaire

Université Alassane Ouattara, Bouaké, Côte d'Ivoire

\begin{abstract}
Cutaneous leishmaniasis is a parasitosis caused by protozoa of the genus Leishmania and is widespread worldwide. A medical survey carried out in three villages of the city of Bouaké, around suspected cases of cutaneous leishmaniasis, confirmed the presence of this pathology in these villages. As part of the identification of the actors of the epidemiological cycle of cutaneous leishmaniasis, an entomological study was conducted in these villages in order to identify potential phlebotomus vectors of leishmaniasis. Oil traps and CDC light traps were set between March and June 2019,
\end{abstract}


following a rotation method between trapping sites. They were set between 5:00 pm and 6:00 pm and were collected the next morning between 7:00 am and 8:00 am. The specific diversity of the identified species was determined from the ecological indices of equitability and Hill's ecological indices. These traps allowed the capture of 135 sandfish, 78 of which were morphologically identified. The genus Sergentomyia constituted $91 \%$ of our harvests against 9\% for the genus Phlebotomus. Ph. bergeroti, Ph. rodhaini and Ph. sergenti were the species of the genus Phlebotomus caught at these sites. Further work on the identification of the parasite in both sandflies and humans should be carried out in order to identify the sandflies incriminated in the transmission of leishmania in Bouaké.

Keywords: Cutaneous leishmaniasis, Phlebotomus, Sergentomyia, Bouaké, Côte d'Ivoire

\section{Introduction}

Les phlébotomes, aussi appelés « mouches des sables », sont des insectes diptères nématocères de petite taille dont seule la femelle est hématophage. Sur environ 70 espèces suspectées vectrices, une vingtaine seulement sont des vecteurs prouvés d'espèces anthropotropiques de Leishmania regroupées en 5 à 24 genres selon les auteurs (Lawyer \& Perkins, 2000 ; Depaquit et al., 2008).

Parmi ces genres, Phlebotomus et Lutzomyia présentent un intérêt médical parce qu'ils sont considérés comme des vecteurs de maladies étiologiquement différentes comme des arboviroses (fièvre à papatasi, virus Toscana), la maladie de Carrion (bartonellose causée par Bartonella bacilliformis) et les leishmanioses provoquées par des protozoaires appartenant au genre Leishmania respectivement dans l'ancien et le nouveau monde (Ihem, 2015).

En Côte d'Ivoire, des cas cliniques de leishmanioses cutanée ont été décrits par différents auteurs (Héroin et Oro, 1967 ; Ouhon et al., 1982 ; Eholié et al., 1985 ; Koné et al., 1986 ; Kouassi et al., 2005). En 2018, 20 prélèvements ont été réalisés sur des personnes ayant des lésions évocatrices de la leishmaniose cutanée dans les villages de Boblénou, Kouadio-Miankro et Golikro par une équipe pluridisciplinaire composée d'agents du district sanitaire de Bouaké nord-est et des chercheurs de l'Institut Pierre Richet de Bouaké après observation des cas suspects de la leishmaniose cutanée provenant de ces villages. Sur ces 20 cas suspects, 9 cas ont été confirmés par biologie moléculaire avec l'identification des leishmanies dans les prélèvements par l'Instituto Salud Carlos III de Madrid, centre de référence de l'OMS (communication personnelle). 
Afin de proposer une stratégie globale de lutte contre la maladie dans ces villages, il est nécessaire d'identifier les acteurs du cycle épidémiologie. Cela a conduit à mener cette enquête entomologique qui visait à identifier les différentes espèces de phlébotomes présentes, leur diversité et leur répartition dans ces localités.

\section{Matériel et méthodes}

\section{Zone, type et durée de l'étude}

Il s'agit d'une étude transversale réalisée dans trois villages situés dans la commune de Bouaké, au centre de la Côte d'Ivoire (figure 1). Elle s'est déroulée de Mars à Juin 2019, soit une durée de 4 mois. La ville se caractérise par un climat de type équatorial de transition. Selon Bagnouls et Gaussen (1957), un mois sec est un mois où les précipitations sont inférieures ou égales à deux fois la température moyenne mensuelle. Ainsi, les mois de Mars et Avril sont des mois à tendance sèche et les mois de Mai et Juin sont des mois à tendance humide (figure 2).

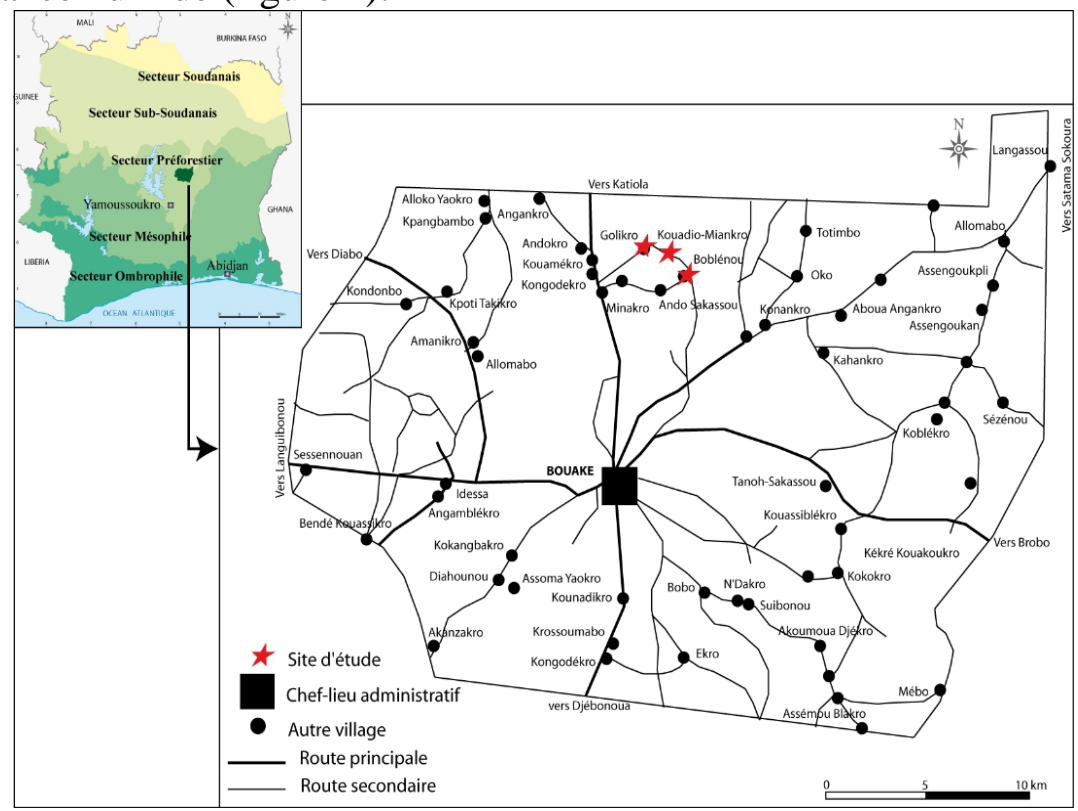

Figure 1 : Zone d'étude présentant les trois villages de la commune de Bouaké, au centre de la Côte d'Ivoire 


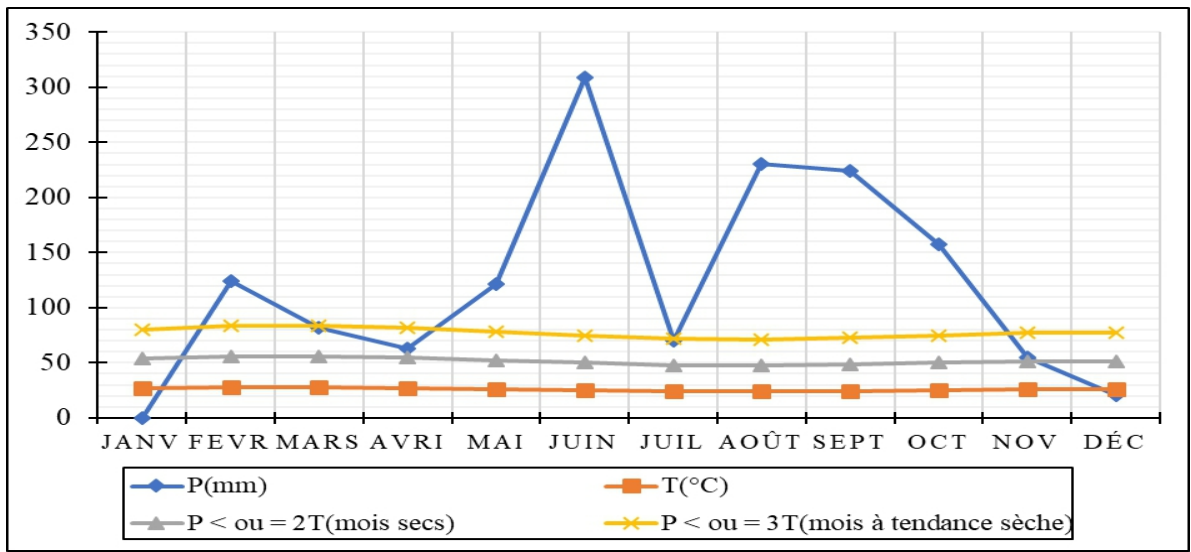

Figure 2 : Evolution des précipitations au cours de l'année 2018 à Bouaké (source : Sodexam)

\section{Technique de piégeage et biotopes inventoriés}

Deux types de pièges ont été utilisés pour la capture des phlébotomes : le piège lumineux CDC et le piège huileux (figure 3). Ces pièges ont été posés dans 13 sites de piégeage répertoriés, géoréférencés et répartis dans les trois villages (6 sites à Boblénou, 4 sites à Golikro et 3 sites à Kouadio-Miankro).
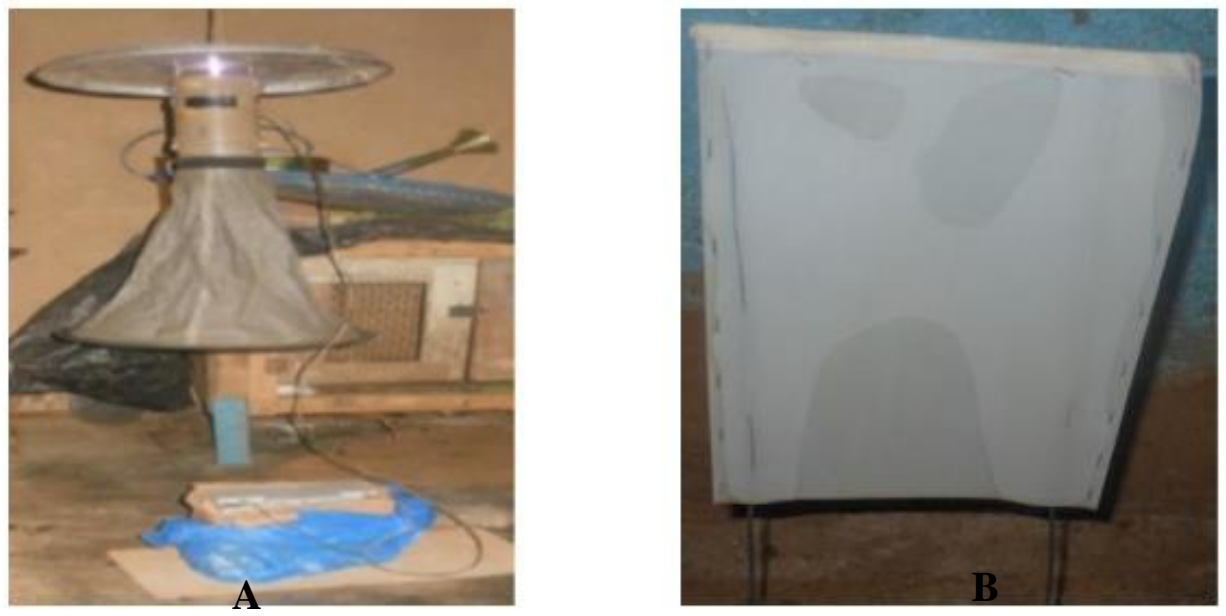

Figure 3 : Piège lumineux $\mathrm{CDC}(\mathrm{A})$ et piège huileux $(\mathrm{B})$

Les pièges ont été posés dans différents biotopes (dépotoirs, intérieur d'habitation et anfractuosité d'arbre) (figure 4) suivant une méthode de rotation. Ils ont été posés entre $17 \mathrm{~h}$ et $18 \mathrm{~h}$ et relevés le lendemain matin entre $07 \mathrm{~h}$ et $8 \mathrm{~h}$. La fréquence des captures était de trois jours par semaine pendant la période de mars à juin 2019.

Les spécimens capturés par les pièges huileux ont été conservées dans de l'alcool à $70^{\circ}$ avant d'être éclaircis dans le liquide de Mac-André pendant $48 \mathrm{~h}$, puis dans une solution de potasse à $15 \%$ pendant $24 \mathrm{~h}$. Ils ont ensuite été 
remis dans le liquide de Mac-André pendant 24 h et enfin montées dans de l'alcool polyvinylique et identifiés sur la base de leurs caractères morphologiques.

Quant aux spécimens capturés par les pièges lumineux CDC, ils ont été directement éclaircis dans le liquide de Mac-André pendant 48h, puis dans la solution de potasse à $15 \%$ pendant $24 \mathrm{~h}$, ensuite dans le liquide de MacAndré pendant $24 \mathrm{~h}$ et enfin montées dans de l'alcool polyvinylique avant d'être identifiés.

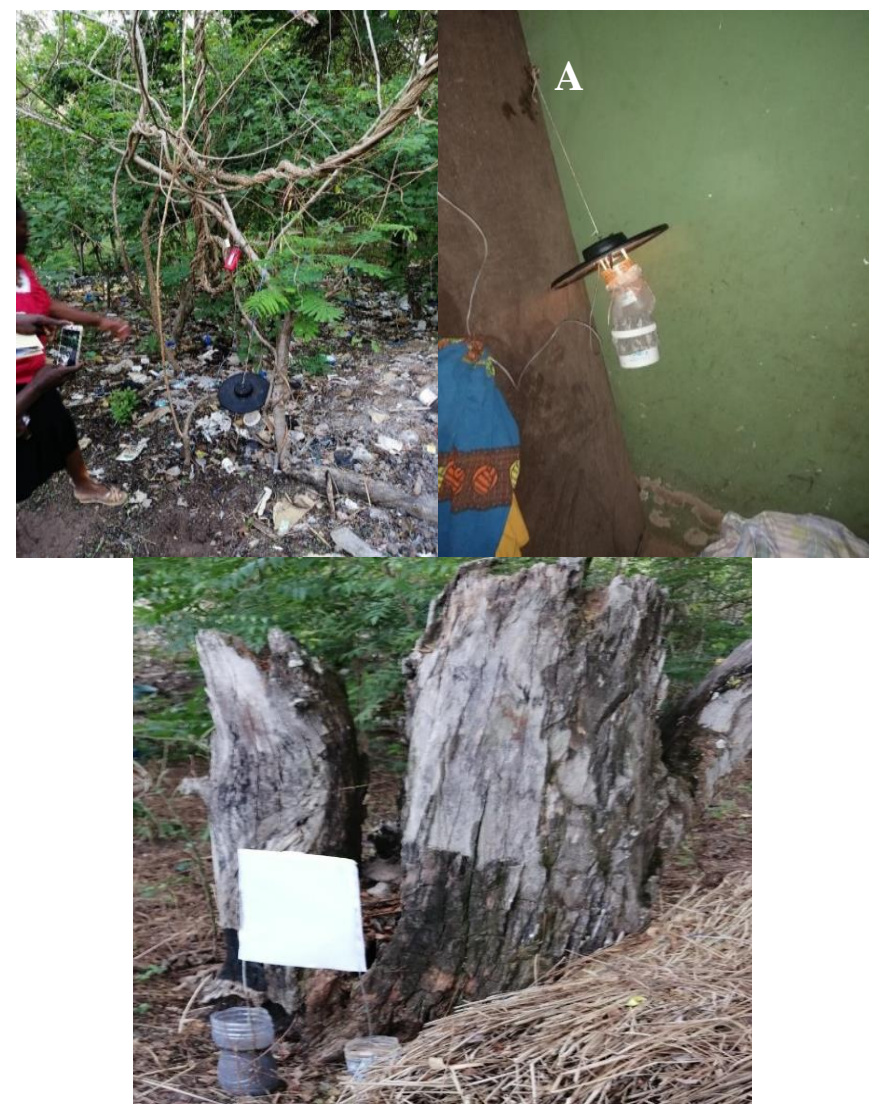

Figure 4 : Biotopes prospectés (A : dépotoir, B : intérieur d'habitation, C : anfractuosité d'arbre)

L'identification des phlébotomes s'est faite morphologiquement sous une loupe binoculaire à partir du logiciel d'identification réalisé par Niang et al (2000), au laboratoire d'entomologie de l'Institut Pierre Richet (IPR) à Bouaké.

\section{Analyse des données}

Les paramètres tels que la richesse spécifique (nombre d'espèces que compte un peuplement), l'abondance (nombre relatif des individus de chaque espèce par rapport à l'ensemble des phlébotomes récoltés) et la fréquence 
relative $(\boldsymbol{F R})$ ou abondance relative (pourcentage d'individus d'une espèce (ni) par rapport au total des individus recensés $(\boldsymbol{N})$ d'un peuplement) ont été déterminés (Dajoz, 1982 ; Ba et al., 1998).

A ces différents paramètres, s'ajoutent les indices écologiques que sont l'indice d'occurrence ou degré de présence (C) qui correspond au nombre de sites contenant l'espèce (i) par rapport au nombre total de site. Cet indice est exprimé en pourcentage. On en distingue cinq classes, si $\mathrm{C}=0 \%-20 \%$ cela signifie que le degré de distribution est sporadique, de $20,1 \%$ à $40 \%$ la distribution des espèces est rare.

Lorsque l'occurrence varie de $40,1 \%$ à $60 \%$ la présence des espèces est modérée, les espèces sont fréquemment distribuées lorsque la valeur est de 60,1 à $80 \%$. Le degré de distribution est constant si $\mathrm{C}=80,1 \%-100 \%$ (Zeroual, 2017).

L'indice d'équitabilité (E) qui mesure la répartition des individus au sein des espèces indépendamment de la richesse spécifique, représente le rapport de l'indice de Shannon-Weaver $\left(\mathbf{H}^{\prime}\right)$ à l'indice maximal théorique dans le peuplement (H' max). Cet indice varie entre 0 (signifie la dominance d'une espèce) et tend vers 1 (toutes les populations d'espèces sont équitablement distribuées) (Spellerberg \& Fedor, 2003).

L'indice de diversité de Hill mesure l'abondance proportionnelle en associant les indices de Shannon-Weaver (H') et de Simpson (D).

Les analyses statistiques sont réalisées avec le logiciel $\mathrm{R}$ version 3.5.1 et avec un risque $\alpha$ de $5 \%$.

\section{Résultats}

Un total de 135 phlébotomes a été capturé au cours de cette étude, dont 78 ont été identifiés. Le village de Boblénou a enregistré une richesse totale de 100 phlébotomes, avec une valeur maximale de 45 phlébotomes dans les anfractuosités d'arbre, et une valeur minimale de 22 phlébotomes au niveau des dépotoirs. A Golikro, la plupart des phlébotomes (23) ont été capturés dans les anfractuosités d'arbre, pendant que le plus faible nombre (1 spécimen) a été capturé à l'intérieur d'habitation. A Kouadio-Miankro, par contre, le maximum de phlébotomes ( 5 spécimens) a été capturé au niveau des dépotoirs (Figure 5).

Sur les 78 phlébotomes identifiés, il y avait 38 mâles pour 40 femelles avec un sex-ratio $(\mathrm{M} / \mathrm{F})$ de 0,95 . 


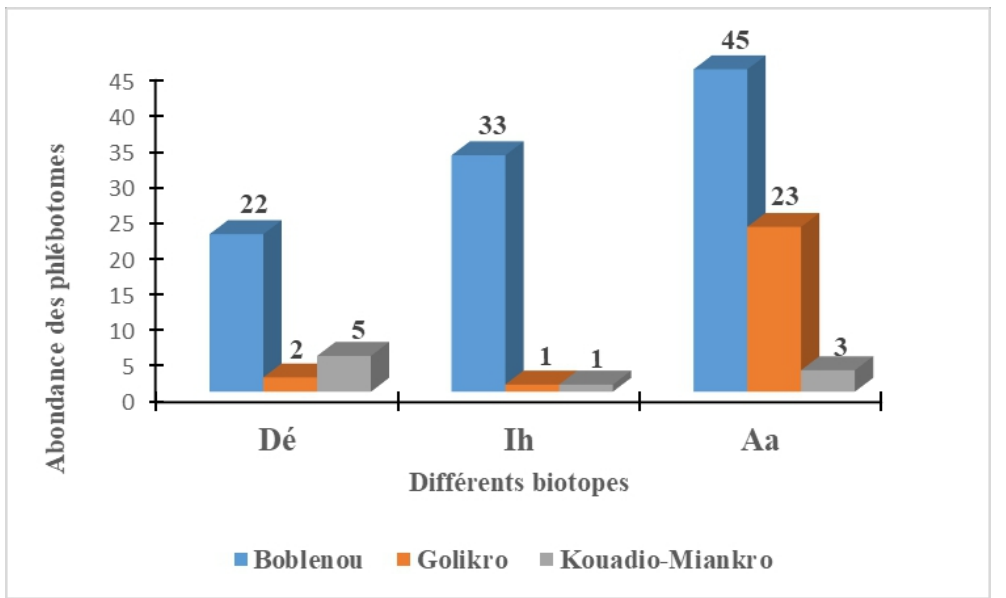

Figure 5 : Richesse totale par biotopes et par village

L'étude systématique de 78 phlébotomes, basée sur les critères morphologiques (Niang et al, 2000) a permis d'identifier 16 espèces appartenant aux genres: Sergentomyia et Phlebotomus. Le genre Sergentomyia (91\%) était le plus représenté de la population de phlébotomes contrairement au genre Phlebotomus (9\%).

L'identification des espèces a permis de noter une richesse spécifique plus importante à Boblénou (14 espèces) par rapport à Kouadio-Miankro (7 espèces) et Golikro (6 espèces) (tableau I).

Tableau I : Richesse spécifique par villages

\begin{tabular}{|c|c|c|c|}
\hline $\begin{array}{l}\text { Villages } \\
\text { Espèces }\end{array}$ & Boblénou & Golikro & Kouadio-Miankro \\
\hline Ph. bergeroti & + & & \\
\hline Ph. rodhaini & + & & \\
\hline Ph. sergenti & + & & \\
\hline Se. adami & + & & \\
\hline Se. adleri & + & & + \\
\hline Se. africana & + & + & + \\
\hline Se. ingrami & + & + & \\
\hline Se. inermis & + & + & + \\
\hline Se. affinis vorax & + & & \\
\hline Se. dureni & + & & \\
\hline Se.decipiens & & + & + \\
\hline Se. congolensis & + & & \\
\hline Se. schwetzi & + & + & + \\
\hline Se. simillima & & & + \\
\hline Se.ghesquierei & + & & + \\
\hline Se.squamipleuris & + & + & \\
\hline
\end{tabular}


L'abondance des phlébotomes au niveau des pièges lumineux CDC (97 spécimens) était plus importante que celle des pièges huileux (38 spécimens). Le test de Wilcoxon utilisé pour comparer l'abondance des phlébotomes entre ces pièges a montré que les pièges lumineux étaient plus attractifs que les pièges huileux $(\mathrm{p}=0,026)$.

La comparaison de l'abondance des phlébotomes entre les villages a été faite avec le test de Parwise Wilcox. Les valeurs de P-value sont mentionnées dans le tableau II.

Tableau II : Comparaison 2 à 2 de l'effectif des phlébotomes capturés

\begin{tabular}{ccc}
\hline Villages & Effectifs & P-value \\
\hline Boblénou-Golikro & $100-26$ & $\mathbf{0 , 0 3}$ \\
Boblénou-Kouadio-Miankro & $100-9$ & $\mathbf{0 , 0 0 0 6}$ \\
Golikro-Kouadio-Miankro & $26-9$ & 0,28 \\
\hline
\end{tabular}

Selon les valeurs d'occurrence, les résultats montrent clairement la présence de 4 classes (Tableau III).

Il s'agit de la classe des espèces fréquemment distribuées $(\mathrm{C}=$ $61,54 \%)$, celle des espèces distribuées modérément $(C=46,15 \%)$, celle à distribution rare $(\mathrm{C}=23,08 \%)$ et celle des phlébotomes à distribution sporadique $(\mathrm{C}<20 \%)$.

Tableau III : Classes d'occurrence des phlébotomes identifiés au cours de l'étude

\begin{tabular}{lccc}
\hline \multicolumn{1}{c}{ ESPECES } & $\mathrm{C} \%$ & $\begin{array}{c}\text { CLASSE } \\
\text { D'OCCURRENCE }\end{array}$ & $\begin{array}{c}\text { NOMBRE DES } \\
\text { ESPECES }\end{array}$ \\
\hline Se. africana & 61,54 & fréquente & 2 espèces \\
Se. schwetzi & 61,54 & fréquente & \\
Se. squamipleuris & 46,15 & modérée & 1 espèce \\
Ph. bergeroti & 23,08 & rare & \\
Se. inermis & 23,08 & rare & 2 espèces \\
Ph. rodhaini & 15,38 & sporadique & \\
Ph. sergenti & 7,69 & sporadique & \\
Se. adami & 15,38 & sporadique & \\
Se. adleri & 15,38 & sporadique & \\
Se. ingrami & 15,38 & sporadique & \\
Se. affinis vorax & 7,69 & sporadique & 11 espèces \\
Se. dureni & 7,69 & sporadique & \\
Se. decipiens & 15,38 & sporadique & \\
Se. congolensis & 7,69 & sporadique & \\
Se. simillima & 15,38 & sporadique & \\
Se.ghesquierei & 15,38 & sporadique & \\
\hline
\end{tabular}

Les valeurs des indices de Shannon-Weaver (H'), de Simpson (D), de l'équitabilité de Pielou (E), de l'indice de Hill et l'opposé de l'indice de Hill (1-Hill) ont été déterminées par saison. 
L'abondance la plus élevée des phlébotomes identifiés a été obtenue au cours de la période sèche, avec une abondance globale de 55 individus et une richesse spécifique de 14 espèces. Les indices de diversité calculés au cours de ces mois (Mars-Avril) à tendance sèche étaient proches de $1 \mathrm{ou}$ supérieure à 1 (figure 5) montrant ainsi une grande diversité des phlébotomes en période sèche. Sur 23 individus obtenus en période pluvieuse, seulement 8 espèces de phlébotome ont été identifiées.

Les valeurs des indices de Shannon Weaver $(1,64)$, de l'opposé de l'indice de Hill $(0,74)$, de Simpson (0.74) étaient au voisinage de 1 ou au-delà, montrant une diversité des espèces identifiées (figure 6).

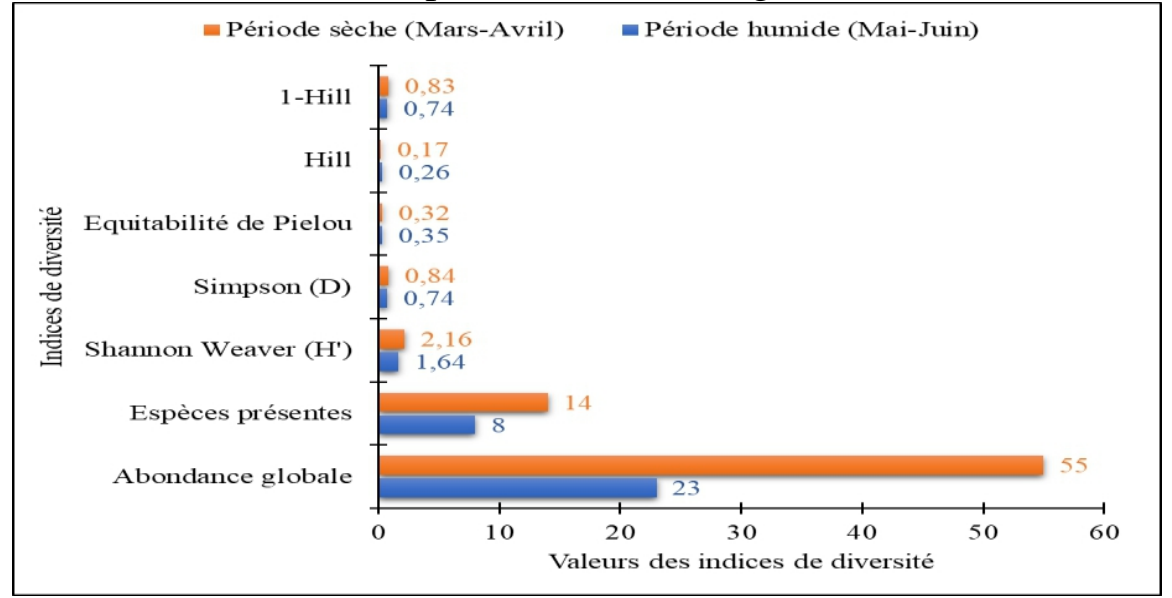

Figure 6 : Indices de diversité selon les saisons.

\section{Discussion}

L'enquête entomologique effectuée dans les villages de Boblénou, Golikro et Kouadio-Miankro a révélé la présence de 3 espèces appartenant au genre Phlebotomus et de 13 espèces appartenant au genre Sergentomyia. Parmi les 3 espèces de Phlebotomus capturées, deux sont considérées comme vecteurs potentiels de la leishmaniose cutanée. Il s'agit de Phlebotomus sergenti et de Phlebotomus bergeroti.

Ph. sergenti est un vecteur prouvé de Leishmania tropica parasite de la leishmaniose cutanée (Killick-Kendrick, 1990). Il a été identifié comme vecteur de Leishmania tropica, dans la province d'Azilal, au Maroc, lors des études réalisées par Ajaoud (Ajaoud et al., 2015) et par Zouirech (Zouirech et al., 2018).

Au cours de la présente étude, Ph. sergenti a été capturé que dans le village de Boblénou avec une faible proportion (1,28\%). Cette faible proportion s'expliquerait par la relative courte durée de notre étude qui était de 4 mois (mars à juin) alors qu'elle était de 50,21\% lors d'un inventaire entomologique au Maroc sur une durée de 8 mois d'avril à novembre 2018 (Zouirech et al., 2018). Aussi, les latitudes et les climats de ces zones 
géographiques (Côte d'Ivoire et Maroc) sont différents. Ces paramètres pourraient avoir un impact sur l'abondance des espèces de phlébotomes vivant en zone de forêt, de savane, de steppes ou de désert.

Phlebotomus bergeroti est une espèce qui selon Trouillet et Faye, peut jouer un rôle épidémiologique important dans la transmission de la leishmaniose cutanée au Sahara central (Trouillet \& Faye, 1993). Elle a été capturé dans le village de Boblénou. Au cours d'une étude de détection moléculaire des parasites de la leishmaniose réalisée en Iran, une femelle de Phlebotomus bergeroti a été révélé infesté par Leishmania major agent de la leishmaniose cutanée (Azizi et al., 2016). Ce qui met en exergue son rôle de vecteur potentiel dans la transmission de la leishmaniose cutanée.

La faible proportion des espèces du genre Phlebotomus peut aussi s'expliquer par le fait que les phlébotomes appartenant à ce genre se rencontrent en majorité dans les régions arides, semi-arides et subtropicales de l'Afrique, de l'Asie et du sud de l'Europe (Killick-Kendrick, 1990).

Les 13 espèces de phlébotomes du genre Sergentomyia identifiées au cours de cette étude ont été capturées dans les trois villages. Parmi elles, 10 font partie des espèces déjà identifiées en Côte d''Ivoire. Des études réalisées au Kenya (Kaddu et al., 1986 ; Mutinga et al., 1994), en Ethiopie (Hailu et al., 1995), au Ghana (Nzelu et al., 2014) et au Sénégal (Senghor et al., 2016) ont trouvé Se. ingrami, Se. schwetzi, Se. affinis vorax, Se. Squamipleuris et Se. africana, porteurs de leishmanies responsables de leishmaniose cutanée. En effet, l'ADN de Leishmania tropica et l'ADN de L. major ont été retrouvé dans Se. ingrami. L'ADN de L. infantum a été isolé de Se. schwetzi et Se. affinis vorax, et celui de L. donovani a quant à lui été observé chez $S e$. squamipleuris, Se. africana, Se. ingrami, et Se. schwetzi. L'on ne peut donc pas exclure totalement le rôle des espèces de Sergentomyia dans la circulation des leishmanies responsables de la leishmaniose cutanée à Bouaké.

Le nombre de phlébotomes femelle était légèrement supérieur à celui des mâles $($ sex-ratio $=0,95)$. La présence d'individus de sexe féminin identifiés met en évidence l'effet de nuisance et l'exposition de la population des villages prospectés aux piqûres de phlébotomes femelles infestées, ce qui entrainerait la propagation de la leishmaniose.

Les captures réalisées lors de cette étude ont permis de montrer que les pièges lumineux CDC étaient plus efficaces pour la capture des phlébotomes que les pièges huileux. Ces résultats sont similaires à ceux trouvés lors d'une étude comparative sur le terrain pour l'évaluation de la performance de trois types de pièges (pièges lumineux $\mathrm{CDC}(24 \%)$, pièges à $\mathrm{CO}_{2}(66,7 \%)$ ) et pièges huileux $(8,4 \%))$ pour la capture de phlébotomes dans le nord-est de l'Italie (Signorini et al., 2013). Bien que l'efficacité des pièges lumineux (24\%) fût intermédiaire, elle était cependant supérieure à celle des pièges huileux $(8,4 \%)$. 
Les espèces de phlébotomes sont inégalement réparties. Certaines espèces ont été retrouvées en grand nombre alors que d'autres ne sont représentées que par de rares spécimens. Toutefois, cette inégalité, s'explique très probablement par la phénologie des espèces durant la période d'étude et aussi les conditions favorables de certains biotopes au développement de certaines espèces. Malgré cette diversité, les phlébotomes ont plusieurs caractères en commun. Ils sont tous nocturnes, se cachent le jour dans des micro-habitats sombres et humides et sont capables de s'introduire dans des espaces confinés pour éviter les conditions extrêmes de température et d'humidité (Kabbout et Merzoug, 2017).

Une variation saisonnière de l'abondance des phlébotomes a été observée, montrant une abondance relativement faible au cours de la saison pluvieuse humide contrairement à la saison sèche chaude aux mois de mars et avril. Ces résultats sont semblables à ceux qui ont déjà été décrits au Mali en 2006 au cours d'une étude sur l'épidémiologie de la leishmaniose cutanée à Kemena et Sougoula avec une grande abondance en saison sèche chaude pour l'ensemble des phlébotomes capturés (Samaké, 2006). Par contre, la diminution des effectifs observée en saison des pluies pourrait être due à la force du vent et les précipitations importantes qui sont néfastes à la survie de ces petits insectes.

L'eau de pluie pourrait effectuer un drainage des œufs et des larves de phlébotomes situés dans le sol, en particulier au niveau des dépotoirs, lieux favorables au développement des œufs et des larves de phlébotomes. Ainsi la pluie constituerait un facteur limitant au développement des œufs et larves de phlébotomes (Omar et al., 2018).

Selon Bigot et Bodo, les indices de diversité sont élevés lorsque les conditions du milieu sont favorables pour l'ensemble des espèces qui y vivent, dans le cas contraire ces indices sont moindres (Bigot L. \& Bodot P., 1973). C'est ce qui a été observé dans cette étude. En effet, les valeurs des indices obtenues pendant les deux saisons étaient au voisinage de 1 ou au-delà, montrant une relative bonne diversité des espèces identifiées. Ceci s'expliquerait par le fait qu'en saison sèche, humide, la température $\left(19-20^{\circ} \mathrm{C}\right)$ et le vent (limite : 1-1,5m) sont propices à leur activité nocturne et crépusculaire (Léger \& Depaquit, 1999).

Également, à cause de la chaleur, les humains se reposent hors de leurs habitations sans se protéger contre les piqûres de phlébotomes femelles. Ce qui donne l'opportunité à ces insectes de prélever aisément le sang nécessaire à la maturation de leurs œufs. Par contre, en saison des pluies, le vent et la pluie étant des facteurs limitants du développement des œufs et des larves, les phlébotomes se réfugient dans les fissures à l'intérieur d'habitation et dans les anfractuosités d'arbres où les conditions de température $\left(19-20^{\circ} \mathrm{C}\right)$ et de vent (limite : 1-1,5m) sont propices à leur activité nocturne et crépusculaire et 
constituent aussi un lieu de protection contre les vents et la dessiccation, de même qu'un lieu de reproduction (Léger et Depaquit, 1999 ; Colange, 2011).

Cette étude aura permis de faire un inventaire de la faune phlébotomienne dans les villages prospectés de la commune de Bouaké, mais aussi de suspecter les espèces du genre Phlebotomus identifiées, comme de potentiels vecteurs de cette maladie.

\section{Conclusion}

L'étude réalisée dans les villages de Boblénou, Golikro et KouadioMiankro a révélé la présence de trois espèces de phlébotomes du genre Phlebotomus et trois nouvelles espèces du genre Sergentomyia. Ces espèces enrichissent la liste des phlébotomes de Côte d'Ivoire. Dans l'Ancien monde, seules les espèces du genre Phlebotomus sont des vecteurs prouvés de la leishmaniose cutanée. Leur présence dans les captures permet de les suspecter fortement comme des vecteurs de la leishmaniose cutanée dans la zone d'étude. Dans la littérature, le rôle vecteur des espèces du genre Sergentomyia est de plus en plus suspecté.

Des études plus approfondies telles que la détermination du parasite à la fois chez les espèces identifiées et dans les prélèvements réalisés dans ces trois villages doivent être réalisées, de même que la détermination de leur capacité vectorielle afin d'incriminer celles vectrices de la leishmaniose cutanée dans la commune de Bouaké.

\section{Remerciements}

Nous tenons à exprimer notre gratitude au District Sanitaire Bouaké nord-est pour nous avoir accordé l'autorisation pour la conduite de ce travail. Nous sommes reconnaissants aux populations, à la chefferie et à la notabilité des villages de Boblénou, Golikro et Kouadio-Miankro pour leur disponibilité à la réalisation de nos travaux de terrains. Nous remercions les techniciens entomologistes de l'Institut Pierre Richet (IPR) de Bouaké pour leur aide dans la réalisation de nos travaux de terrains et de laboratoire.

\section{References:}

1. Ajaoud M, Es-Sette N, Charrel RN, Laamrani-Idrissi A, Nhammi H, Riyad M. \& Lemrani M. (2015). Phlebotomus sergenti in a Cutaneous Leishmaniasis Focus in Azilal Province (High Atlas, Morocco): Molecular Detection and Genotyping of Leishmania tropica, and Feeding Behavior. PLOS Neglected Tropical Diseases 9 (3) e0003687.

2. Azizi K, Askari MB, Kalantari M. \& Moemenbellah-Fard MD. (2016). Molecular detection of Leishmania parasites and host blood meal identification in wild sand flies from a new endemic rural region, south of Iran. Pathogens and global health, 110 (7-8) : 303-309. 
3. Ba Y, Trouillet J, Thonnon J. \& Fontenille D. (1998). Phlébotomes du Sénégal (Diptera-Psychodidae): Peuplement et dynamique des populations de la région de Mont-Rolland. Parasite, 5 (2) : 143-150.

4. Bagnouls F. \& Gaussen H. (1957). Les climats biologiques et leur classification. Annales de Géographie, 66 (355) : 193-220.

5. Bigot L, \& Bodot P. (1973). Contribution à l'étude biocenotique de la garrigue à Quercus coccifera III. Dynamique de la zoocenose d'invertébrés. - Vie Milieu. Ser C Biol Terr 25 (2) : 251-267

6. Colange H. (2011). Contribution à l'étude du repas sanguin de Phlebotomus perniciosus (Diptera : Psychodidae) [PhD Thesis]. Ecole Nationale Vétérinaire de Toulouse- ENVT ; 78p

7. Dajoz R. (1982). Précis d'écologie. Écologie fondamentale et appliquée. Ed. GauthierVillars Paris. 505p

8. Depaquit J, Léger N. \& Robert V. (2008). Les Phlébotomes de Madagascar (Diptera: Psychodidae). VI - Un sous-genre nouveau (Vattieromyia) avec trois espèces nouvelles: Sergentomyia (V.) sclerosiphon, S. (V.) namo et $S$. (V.) anka. Parasite, 15 (1) : 15-26.

9. Eholié SP, Tanon AK, Folquet-Amorissani M, Doukouré B, Adoubryn KD, Yattara A, \& Bissagnéné E. (1985). Trois nouveaux cas de leishmaniose viscérale autochtone en Côte d'Ivoire. Bull Soc Pathol Exot, 101 (1) : 60-61.

10. Hailu A, Balkew M, Berhe N, Meredith SEO. \& Gemetchu T. (1995). Is Phlebotomus (Larroussius) orientalis a vector of visceral leishmaniasis in South-west Ethiopia? Acta Tropica, 60 (1): 15-20.

11. Héroin P, \& Oro J. (1967). Première observation de leishmaniose cutanée en Côte d'Ivoire. Revue Médicale Côte d'Ivoire, 3 (6) : 32.

12. Ihem NBS. (2015). Contribution à l'étude de la biodiversité des Phlébotomes (Diptera: Psychodidae) dans la région de Constantine. [Thèse]. Université des Frères Mentouri Constantine, $77 \mathrm{p}$

13. Kabbout N. \& Merzoug D. (2017). Contribution à l'étude bioécologique des insectes d'intérêt médical dans le nord-est algérien. Th.

Doct. Université Oum El Bouaghi;

République Démocratique et Populaire Algérie, 224p

14. Kaddu JB, Mutinga MJ. \& Nyamori MP. (1986). Leishmania in kenyan phlebotomine sandflies - IV: Artificial feeding and attempts to infect six species of laboratory-reared sandflies with Leishmania donovani. International Journal of Tropical Insect Science, 7 (06) : 731-735.

15. Killick-Kendrick R. (1990). Phlebotomine vectors of the leishmaniasis: A review. Medical and Veterinary Entomology, 4 (1) : 1-24. 
16. Koné M, Ouhon J, Assoumou A, Colin M, Ferly-Thérizol M, \& Assalé G. (1986). Leishmaniose cutanée en Côte d'Ivoire : Étude clinique et parasitologique (à propos de six cas). Revue Médicale Côte d'Ivoire, $75,128$.

17. Kouassi B, Holo K, Achi VH, Adoubryn k, \& Kakou E. (2005). Leishmaniose viscérale à Abidjan à propos de 3 observations. Médecine Tropicale, 65 : 602-603.

18. Lawyer PG, \& Perkins PV. (2000). Leishmaniasis and trypanosomiasis. In Medical Entomology Springer, 231-298.

19. Léger N. \& Depaquit J. (1999). Les phlébotomes. In: Dedet JP, editor. Les Leishmanioses. Paris: Ellipses, 89-108.

20. Mutinga MJ, Massamba NN, Basimike M, Kamau CC, Amimo FA, Onyido AE, Omogo DM, Kyai FM. \& Wachira DW. (1994). Cutaneous leishmaniasis in Kenya : Sergentomyia garnhami (Diptera Psychodidae), a possible vector of Leishmania major in Kitui District : a new focus of the disease. East African Medical Journal, 71 (7): 424-428.

21. Niang AA, Geoffroy B, Angel G, Trouillet J, Killik-Kendrick R, Hervy JP \& Brunhes J. (2000). Les phlébotomes de l'Afrique de l'Ouest. Logiciel d'identification et d'enseignement, IRD édition.

22. Nzelu C O, Kato H, Puplampu N, Desewu K, Odoom S, Wilson MD, Sakurai T, Katakura K. \& Boakye DA. (2014). First detection of Leishmania tropica DNA and Trypanosoma species in Sergentomyia sand flies (Diptera: Psychodidae) from an outbreak area of cutaneous leishmaniasis in Ghana. PLoS neglected tropical diseases, 8 (2): e2630

23. Omar SS, Serigne M, \& Niang AA. (2018). Faune phlébotomienne du foyer leishmanien de Keur Moussa : Données actuelles sur la diversité et variations saisonnières (Thiès, Sénégal). Bulletin de la Société de Pathologie Exotique, 111 (1) : 38.

24. Ouhon J, koné M, Assoumou A, Bleulaine SC, Piguel M, Aka PA, Ferly-Thérizol M. \& Assalé G. (1982). A propos de 3 cas de leishmaniose cutanée humaine diagnostiqués au laboratoire de parasitologie (C.H.U de Cocody-Abidjan). Revue Médicale de Côte d'Ivoire, 188 (67) : 32-37.

25. Samaké S. (2006). Epidémiologie de la leishmaniose cutanée à Kemena et Sougoula (Cercle de Baroueli. Thèse Pharmacie, Université de Bamako 120 p.

26. Senghor MW, Niang AA, Depaquit J, Ferté H, Faye MN, Elguero E, Gaye O, Alten B, Perktas U. \& Cassan C. (2016). Transmission of Leishmania infantum in the canine leishmaniasis focus of MontRolland, Senegal: Ecological, parasitological and molecular evidence 
for a possible role of Sergentomyia sand flies. PLoS neglected tropical diseases, 10 (11): e0004940.

27. Signorini M, Drigo M, Marcer F, di Regalbono A F, Gasparini G, Montarsi F, Pietrobelli M, \& Cassini R. (2013). Comparative field study to evaluate the performance of three different traps for collecting sand flies in northeastern Italy. Journal of Vector Ecology, 38 (2): 374 378.

28. Spellerberg IF, \& Fedor PJ. (2003). A tribute to Claude Shannon (1916-2001) and a plea for more rigorous use of species richness, species diversity and the 'Shannon-Wiener'Index. Global ecology and biogeography, 12 (3) : 177-179.

29. Trouillet J. \& Faye O. (1993). Phlébotomes du Sénégal. Présence de Phlebotomus (Phlebotomus) bergeroti Parrot, 1934 (Diptera, Psychodidae). Annales de Parasitologie Humaine et Comparée, 68 (2) : 101-103.

30. Zeroual S. (2017). Les phlébotomes vecteurs de leishmaniose dans la région de Biskra: Inventaire et écologie des phlébotomes et étude épidémiologique [Thèse]. Université Badji Mokhtar-Annaba,108p.

31. Zouirech M, Rhajaoui M, Faraj C, Guamri Y E, Amahmid O, Hachimi MYE, Bouhout S, Kharrim K E. \& Belghyti D. (2018). Entomological inventory of Phlebotomine sand flies and epidemiological study of leishmaniasis in Afourer, Azilal province, High Atlas, Morocco. Médecine et Santé Tropicales, 4: 385-389. 